\title{
A Survey of Vehicle Routing Problem
}

\author{
Wujun $\mathrm{Cao}^{1}$ and Wenshui Yang ${ }^{2, *}$ \\ ${ }^{1}$ School of Management Engineering, Zhengzhou University, Zhengzhou 450001, China \\ ${ }^{2}$ School of Management Engineering, Zhengzhou University, Zhengzhou 450001, China \\ Corresponding Email: yangwenshui2009@163.com
}

\begin{abstract}
In recent years, vehicle routing problem (VRP) has become an important content in logistics management research, and has been widely used in transportation system, logistics distribution system and express delivery system. In this paper, we discuss the classification of VRP, and summarize the common constraints of VRP, model algorithm and the main research results in recent years. Finally, we analyzes the future of VRP, and it is considered that the intelligent vehicle routing problem and intelligent heuristic algorithm will be an important field of future research.
\end{abstract}

\section{Introduction}

Vehicle Routing Problem was first proposed by Dantzig and Ramsar 1959, and is mainly used to solve the transport route optimization problem: Atlanta's refinery problem. The subject quickly attracted the attention of experts and scholars, such as operations research, management, computer, graph theory, and proved to be widely used in transportation system, logistics distribution system and express delivery system. After several decades of development, the vehicle routing problem has become an important part of logistics management research, and is classified as the general term for such a type of problem: by a number of vehicles from one or more warehouses to multiple geographic On the distribution of customers, how to arrange the vehicle and its route to the total distribution costs can be minimized. Then, in theory, the VRP problem is defined as: organizing a series of loading and unloading points, as well as the corresponding traffic line organization, so that vehicles can be ordered through them. That is, to achieve the objectives and solve certain problems (such as shortest distance, minimum cost, time limit) under certain constraints (goods demand, delivery, delivery time, vehicle capacity constraints, travel restrictions, time constraints, etc.) May be less, the number of vehicles used as little as possible, etc.). From this simple definition, it is easy to see that TSP is a special case of VRP problem, then VRP problem is also a NP problem [1,2]. Shown in Figure 1:

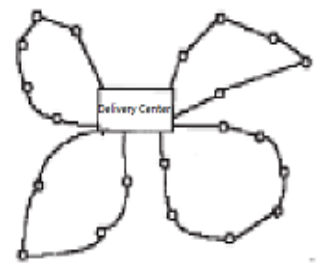

Fig. 1. VPR Schematic diagram. 
At present, the enthusiasm of experts and scholars of the VRP research is still high, and according to different perspectives, the classification of VRP:

(1)According to the number of distribution centers: single distribution center and multi-distribution center problem;

(2)According to the type of vehicle: single-vehicle type and multi-vehicle type problem;

(3)According to the characteristics of the task: pure send (take) cargo problems and loading and unloading mixing problem;

(4)According to whether the time constraints: no time window problem and time window problem;

(5) By vehicle loading: And the problem of non-full load;

(6) According to the optimization of the number of goals: a single objective and multi-objective problem;

(7) Vehicle and vehicle by the ownership of the points: the vehicle open problem and vehicle closure problems;

(8) By mastering the information of certainty: Sexual VRP and non-deterministic VRP problems;

As can be seen from these classifications, solutions to the VRP problem are varied, each category will be mapped a corresponding models and algorithms for solving the natural process is varied. But back to the original point of view to build the model of VRP, VRP problem can be seen as a variant or in combination with integer programming model of graph theory model or other models among which we can find some rules, to achieve the purpose of solving.

\section{Constraints of vehicle routing problem}

As mentioned earlier, VRP problems have a variety of classification methods, for different classification methods also have different corresponding models and algorithms. However, no matter how complex the model of the algorithm, common constraints in the discussion of VRP problem is the same capacity constraints [3]:

(1) Capacity constraints: regardless of which vehicle, it should be less than the total path of the vehicle load capacity. The vehicle routing problem with capacity constraints is brought forward.

(2) Priority constraints: leads to the priority of the vehicle to constrain the path problem.

(3) Vehicle constraints: leads to multi-vehicle vehicle routing problem.

(4) Time window constraints: including hard time window, soft time window constraints. The vehicle routing problem with time window (including hard time window and soft time window) is brought forward.

(5) Compatibility constraints: leads to compatibility constraints of the vehicle routing problem.

(6) Random demand: leads to a random demand for the vehicle routing problem.

(7) Open: leads to open the path of the vehicle problem.

(8)Multi-transport center: leads to a multi-transport vehicle routing problem.

(9)Return transport: leads to a return route with the vehicle routing problem.

(10)The last time period: leads to the vehicle with the last time period issue.

(11Vehicle speed changes with time: with time, leads to the vehicle speed with time changes in vehicle routing problem.

\section{Model and algorithm of vehicle routing problem}

To solve the VRP problem in different classes, you need to find the relationship between the VRPs of different classifications. A simple VRP problem can be solved by building an integer programming model, or by constructing a graph theory model or other related models. Complex VRP problems are only a combination of these models [4].

After analysis, we can extract the following three types from these complex models:(1)vehicle-based model; (2) logistics-based model; (3)multi-coverage model.

After the VRP problem model is set up, the most important step is to solve the model. In recent years, scholars at home and abroad have also proposed many algorithms for obtaining the optimal or suboptimal solution to the different VRP problems. In general, it can be divided into precise algorithms, heuristic algorithms and sub-heuristic algorithms.

\subsection{Accurate algorithm}


In general, it is more efficient to use exact algorithms to compute small-scale VRP problems, and the optimal solution can be solved in a certain time. However, with the increase in the scale of logistics and distribution, it is difficult in a short time to find a satisfactory solution. At present, scholars at home and abroad are not many of the precise algorithm research, including: branch and bound method, cutting plane method, dynamic programming method, network flow algorithm.

(1) Branch and bound method. Branch and bound method is a commonly used, simple and pure integer algorithm for integer programming, mixed integer programming problem. When using this algorithm to solve, we must first set two variables:

A-The problem of Maximizing the integer programming;

B-Linear Programming Problem without Integer Constraints;

If the optimal solution of B does not satisfy the integer condition of A, then the optimal objective function value of B can be regarded as an upper bound of the optimal objective function value $Z *$ of $A$, denoted as $Z$; then the objective function of any feasible solution of $A$ The value can be used as a lower bound of $Z *$, denoted as $Z$. Then we use the adjacency integer of B's non-integer solution as the additional condition to get two branches or two subproblems. In the feasible region of the two subproblems, we can find all the satisfactory solutions of the original integer programming problem. Finally, after continuous branching, and gradually reduce the $Z, Z$ increased, the final will be able to obtain $Z$ *

(2) Cutting plane method. The cutting plane method was first proposed to solve the integer integer programming problem. When solving this problem, it should be noted that no matter what condition (which is often set as the relaxation problem), it is first determined whether the solution is an integer optimal solution, and if so, the operation is stopped. If not, add a new constraint, the purpose is to not meet the conditions before the non-integer solution cut off. After a time of cutting, all the integer solution retained. So that in the end will form an integer point coordinates, that is, the original integer optimal solution.

(3) Dynamic programming method. Dynamic programming method is a kind of optimization algorithm commonly used in system analysis to solve multi-stage decision process problem. This method is to ensure the dynamic nature of the planning process, that is, at every stage to achieve system optimization. Any complex problem can be divided, and can be classified after segmentation, each category in the planning problem in the position occupied is not the same, we need to do is to ensure that these different categories occupy the position of the most optimization. And then through a step by step recursive operation, so that each stage can achieve the best decision-making and choice, so that the final can achieve global optimal.

(4) Network flow algorithm. Network flow algorithm is an algorithm to solve small-scale VRP problem, because the network model of the algorithm vertex number and the number of sides, will significantly affect the network flow algorithm time efficiency. Therefore, in the construction of VRP network model, to find the network need to adjust the largest arc; then, by adjusting the arc flow and potential, making the need to adjust the arc flow reduction until the network flow is zero.

\subsection{Heuristic algorithm}

Heuristic algorithm has been used to solve the VRP research, now talked about more: saving method, two-stage method, scanning method.

(1) Saving method. The conservation law was proposed by Clark and Wright in 1964 to solve the problem of VRPs where the number of vehicles is not fixed. The basic idea of the saving algorithm is that the path is sorted according to the size of the saving value, and the corresponding two customer points are discharged into the path in the order of the capacity of the vehicle and in the order of big to small until all the customers can be discharged. Into the path. Solomon in 1983, the law will be applied to solve the problem with the time window constraints VRP. At this time, to consider the savings in the two customers into the path of the larger, but also consider the time window restrictions. The advantage of the saving algorithm is that it has a faster calculation speed, especially for small-scale distribution optimization, the optimization accuracy is very close to the optimal solution, and can be obtained in a short time. The disadvantage is that with the increase in the size of customers, optimization results will be some deviation. 
(2) Two-stage method. Two-stage method is mainly used to solve the shortcomings of the construction algorithm, it can be said that the two-stage heuristic algorithm for an improvement. In the first stage, the algorithm is used to construct a heuristic algorithm, and then a feasible solution can be obtained. In the second stage, Insert method, 2 - Exchange, 2-swap, 2-OPT, 3-OPT and other methods to improve the technology to ensure the feasibility of the solution, through the adjustment of the point to achieve the goal of optimization results. The main goal of this algorithm is to replace the existing solution of the objective function with the feasible solutions generated in each stage, and to get closer to the optimal solution step by step, and finally to optimize the objective function. Therefore, using the two-stage method, the interactive optimization technique can be used to effectively combine the subjective active action with the VRP problem to solve the problem quickly and easily.

(3) Scanning method. The scanning method was proposed by Gillette and Miller. The method is proposed to solve the problem of vehicle routing problem. In the use of scanning method, the first point to be divided about the location of a demand point, where the polar coordinates are often used; and then set any point corresponding to the point of zero polar coordinates, that is, as the starting point, on this basis, The required service range of clockwise or counterclockwise to divide the division of the restriction is the car capacity; Finally, the use of Lin and Kernighan exchange of previously set demand points sorted, and ultimately to the establishment of the vehicle row Process line. Scanning method is not complicated to use, but the need to ensure the accuracy of program editing.

\subsection{Heuristic algorithm}

The heuristic algorithm, also known as the intelligent heuristic algorithm, uses the heuristic algorithm to replace the decision criterion of the precise algorithm, so it can effectively narrow the search space. Since the 1990s, artificial intelligence has shown some powerful functions in the field of production, especially when solving combinatorial optimization problems, its intelligence is particularly prominent. At present, it is common to introduce artificial intelligence into the solution of VRP problem, and construct an intelligent heuristic algorithm. Commonly include: genetic algorithm, simulated annealing algorithm, tabu search algorithm, ant colony algorithm [5].

(1) Genetic algorithm. The earliest use of genetic algorithms for VRP research is J. Lawrence, and also designed to effectively solve the problem with the time window of the VRP. As the traditional genetic algorithm is a large-scale, coarse-grained optimization algorithm. Therefore, Barnier combines it with the constraint satisfaction problem, and uses the genetic algorithm to deal with the subdomain of the parameter which satisfies the constraint satisfaction problem (the fitness of the gene can be obtained by calculating the solution of CSP), which can reduce the search space and reduce the constraint satisfaction The objective function of the problem and the complexity of the genetic algorithm constraints. Some scholars use genetic algorithm to ensure the global search, with 3-OPT algorithm to enhance the local search capabilities, to solve the VRP joint algorithm. This hybrid algorithm can now be used to solve large-scale VRP problems (about 199 customers).

(2) Simulated annealing algorithm. The simulated annealing algorithm is a stochastic optimization algorithm based on the Monte-Carlo iterative method. The basic idea is mainly derived from the solid annealing principle. The solid is first heated to a sufficiently high temperature and allowed to cool slowly. Because the particles in the solid state become disordered with the temperature when they are heated, then the internal energy increases, but after the slow cooling, the particles gradually become orderly state, in which each temperature Are maintained in equilibrium, so that the reference state at room temperature, the internal energy will be reduced to a minimum. According to the Metropolis criterion, the probability of the particle tends to equilibrium at temperature $\mathrm{T}$ is: e $(-\Delta \mathrm{E} /(\mathrm{kT}))$, where $\mathrm{E}$ is the internal energy at temperature $\mathrm{T}, \Delta \mathrm{E}$ is the change, and $\mathrm{k}$ is Boltzmann's constant. With solid annealing simulation combination optimization problem, the internal energy E can be modeled as the objective function value $\mathrm{f}$, the temperature $\mathrm{T}$ evolved into the control parameter $t$, that is, the simulated annealing algorithm to solve the combinatorial optimization problem: the initial solution $i$ and the control parameter initial value $t$ At the end of the algorithm, the current solution is the approximated optimal solution, which is based on the Monte Carlo Iteration (iterative method), which is based on Monte Carlo Iteration A Heuristic Stochastic Search Process for Solving. The annealing process is governed by a cooling 
schedule consisting of the initial value of the control parameter $t$ and its attenuation factor $\Delta t$, the number of iterations $\mathrm{L}$ for each $t$ value, and the stopping condition $\mathrm{S}$. To sum up, the simulated annealing algorithm can effectively avoid the optimization of the serial structure which is local minimally and eventually tends to the global optimum by giving the search process a time-varying and finally to zero probability jumping.

(3) Tabu search algorithm. The first tabu search algorithm proposed by Glover, mainly to solve the problem of global optimization step by step. When tabu search algorithm is used, it always starts with an initial feasible solution, after a series of specific search test behavior, and finally chooses the behavior that can change the most value of a particular objective function value. In order to avoid the dilemma of local optimal solution, tabu search algorithm uses a flexible "memory" technology, the process has been recorded and selected in order to guide the direction of the next search, saving time, Improve efficiency.

(4) Ant colony algorithm. Ant colony algorithm is a natural phenomenon of the algorithm, has a strong anti-biochemical capacity. The algorithm was first proposed in 1992, inspiration is to observe the ants in the path of food to find inspiration. Ant colony in the process of looking for food, there is a particularly important volatile substances, called pheromones, ant can also be said to find a variety of food behavior, are under the guidance of this pheromone. Ants in the ant can be released according to the companion pheromone concentration, to determine their own forward direction will be. You know, each ant will walk through a road when the release of pheromones, it can be said that when the concentration of a particular location pheromone exceptionally high, it should be the region where the highest concentration of food. We refer to the problem of the path, that is, the optimal objective function value, the optimal path. Of course, when using this algorithm will have some drawbacks, because the ant colony and ant colony is still relatively scattered before, a single ant colony to find the optimal food concentration may be due to geographical constraints, and mistakenly believe that the local food Is the most, quoted into our equation solving process, that is, we may fall into the local optimal dilemma.

But on the whole, the advantage of ant colony algorithm is greater than the disadvantage, in some of the requirements of the accuracy of the situation is not high, you can quickly solve the problem. Of course, for some complex problems, you can set some restrictions, optimize some parameters, the structure of some of the necessary processes, but also to achieve good results. In recent years, in solving the problem of parameter optimization design of PID controller, the results of ant colony algorithm design and the result of genetic algorithm design are simulated and compared. The results show that the ant colony optimization algorithm has shown a new Effectiveness and application value.

\section{Research prospects}

Logistics distribution cost is one of the main sources of logistics costs, and logistics and distribution costs to a large extent by the arrangement of vehicles and routes to decide. This paper first introduces the classification of vehicle routing problem, and then summarizes the main research results of VRP algorithm in recent years according to the classification of VRP algorithm [6,7]. The research shows that, with the expansion of the scale of logistics distribution, the research of exact algorithms has little significance. In the future for a long time, construct the approximate algorithm, especially the intelligent heuristic algorithm will be the important content of VRP algorithm research. The rapid development of social economy, consumer demand for quality of service is also progress, logistics, as a producer services industry, the importance of this area should be increased. VRP problem can be said to be the key link in the process of logistics system optimization, more can not exist slackness psychology. Faced with such a fierce market competition, we should also pay attention to the competition among logistics companies can say who can make full use of existing information technology and Internet technology, who will be able to rapid development. Therefore, in the future, the alliance vehicle scheduling problem between enterprises will also be a new application research field of VRP problem. 


\section{References}

1. Shi Chun-yan, Huang Hui. Vehicle Routing Problem: Research Summary and Prospect. Logistics Technology, 2014 (12): 75-77.

2. Chen Wen-lan, DAI Shu-gui. Review on the algorithm of vehicle routing problem.Journal of Chuzhou University, 2007 (3): 19-25.

3. Liu Yun-Zhong, XUAN Hui-Yu. Review of Models and Algorithms for Vehicle Routing Problem. Chinese Journal of Management, 2005 (1): 124-130.

4. HAN Juan-juan, LI Yong-xian. Review of dynamic vehicle routing problem. Green Science and Technology, 2015 (5): $285-288$.

5. Fang Jincheng, Zhang Qi-shan. VRP routing algorithm for vehicle routing problem. Journal of Shenyang Institute of Engineering, 2006 (4): 357-360.

6. Li Xiang-yong, Tian Peng. Vehicle routing problem model and algorithm research. Shanghai Jiaotong University Antai College of Economics and Management, 2007 (9).

7. Xie Binglei. Multi-loop optimization strategy for stochastic vehicle routing problem .Systems Engineering -Theory \& Practice, 2007 (9): 167-171. 